\title{
Tardive dyskinesia: Risk factors, prevention, and treatment
}

\author{
Subramoniam Madhusoodanan ${ }^{1 *}$ and Michael J. Spatcher ${ }^{2}$ \\ ${ }^{1}$ Department of Psychiatry, St John's Episcopal Hospital, USA \\ ${ }^{2}$ PGY-III Psychiatry Resident, Department of Psychiatry, St John's Episcopal Hospital, USA
}

\begin{abstract}
Background: Tardive dyskinesia is a complication of antipsychotic treatment characterized by chorieform involuntary movements affecting commonly the orofacial and buccolingual regions, but also trunk and extremities. Even though the exact etiology is not clearly understood, it is believed that upregulation of postsynaptic dopamine receptors after chronic dopamine blockade and neuronal oxidative damage may be implicated. The symptoms may be lifelong in some patients. Risk factors include advanced age, female sex, type of antipsychotic agents and routes of administration, pre existing movement disorders and general health of the patient. Multiple agents including vitamin B6, branched-chain amino acids, Ginko biloba, medications including beta blockers, ondansetron and benzodiazapines have been tried in the treatment of tardive dyskinesia without much success. The newly approved medications valbenazine and deutetrabenazine offer hope to these patients who otherwise had to live with this socially and functionally disabling disorder.
\end{abstract}

Methods: Literature review was conducted using keywords tardive dyskinesia, risk factors, pathophysiology, treatment, Valbenazine, and deutetrabenazine. Search engines used include Pubmed, Cochrane Review, PsycINFO, and Psychiatry Online.

Results: We have summarized the history, pathophysiology, risk factors, and management of TD including the recently approved medications.

Conclusion: Tardive dyskinesia is a disabling, long term side effect of antipsychotic use. Many risks factors predispose patients to the development of symptoms. Until recently, there were no FDA approved treatments. The newly approved medications valbenazine and tetrabenazine have shown promising results for the treatment of tardive dyskinesia.

\section{Introduction}

With the development of neuroleptic drugs, psychiatry finally had reliable medication options to treat psychotic disorders. As with most medical developments, there were downsides to these medications. Several side effects were immediately apparent. However, some side effects were more insidious. Since its discovery in the 1960's, tardive dyskinesia (TD) has been a challenging complication of antipsychotic treatment. Characterized by choreiform involuntary movements, tardive dyskinesia can cause severe distress in the life of a patient, as well as a treatment conundrum for clinicians. Most commonly seen in the oral and facial regions with notable tongue protrusions and fasciculations as well as grimacing, tardive dyskinesia symptoms can affect nearly any part of the body. While the pathophysiology of the syndrome is not clearly understood, evidence has emerged which suggests possible causes. Upregulation of postsynaptic dopamine receptors, after chronic dopamine blockade, is a likely causative factor. In addition, neuronal oxidative damage may also be associated with the symptoms. Most alarmingly, the symptoms, even with treatment and withdrawal of the offending agent, can last for years, and may possibly be lifelong.

There have been multiple risk factors postulated to be associated with the development of tardive dyskinesia. Demographics, including patient's age, and female sex are important risk factors. As one would expect, the choice of antipsychotic agent has significant impact on the development and severity of symptoms. However, other factors involved in antipsychotic treatment, including administration schedules, may play a role. General health of the patient, including fasting glucose and pre-existing movement disorders can also predispose the patient to these symptoms.
Given the severity and persistence of the syndrome, several attempts have been made to treat tardive dyskinesia. These treatments include several natural compounds, including vitamins and amino acids, and traditional pharmaceutical therapies. Recently, for the first time, two medications valbenazine and deutetrabenazine were approved by the United States Food and Drug Administration (FDA) for treatment of tardive dyskinesia.

\section{Pathophysiology}

There appears to be an imbalance between dopamine overactivity and cholinergic underactivity [1] This is supported by the observation that patients improve initially with increasing dopamine blockade, and symptoms worsen with administration of anticholinergics. Long term antagonism of postsynaptic dopamine receptors creates a clinical picture resembling that of efferent neuronal destruction. This results in an increased concentration of post synaptic dopamine receptors [2] leading to hypersensitivity to dopamine, and to symptoms of tardive dyskinesia [3].

In addition to the most accepted theory that the super sensitivity to dopamine is the cause of tardive dyskinesia, other factors may also be at play. Christensen, et al. [4] found neuronal loss in the basal ganglia of patients with tardive dyskinesia. This may be due to the fact that

${ }^{\star}$ Correspondence to: Subramoniam Madhusoodanan, Department of Psychiatry, St John's Episcopal Hospital, 327 Beach 19th Street, Far Rockaway, NY 11691 USA, E-mail: sdanan@ehs.org

Received: August 29, 2019; Accepted: September 13, 2019; Published: September 18, 2019 
D2 blockade can increase the release of glutamate and aspartate [5] Glutamate, if chronically activated, has in turn been shown to cause neuronal damage [6]. Tsai, et al. [7] showed that patients with tardive dyskinesia had higher concentrations of excitatory neurotransmitters, including $\mathrm{N}$-acetylaspartate, $\mathrm{N}$-acetylaspartylglutamate, and aspartate in cerebrospinal fluid (CSF) analysis compared to patients without TD symptoms.

It has been found that, among schizophrenic patients, Abnormal Involuntary Movement Scale [8] (AIMS) scores were correlated with higher $\mathrm{N}$-acetylaspartate, $\mathrm{N}$-acetylaspartylglutamate, aspartate, and glutamate CSF concentrations. An inverse relationship existed between AIMS score and superoxide dismutase activity, suggesting that not only do patients with abnormal movements have a higher concentration of neurotransmitters that cause oxidative stress, but also have a decrease ability to cope with oxidative stress. These trends were noted as a whole among patients with schizophrenia, and there was no correlation of these markers and AIMS scores specifically within the TD patient group [7].

A particularly concerning aspect of tardive dyskinesia is the long term possibly lifetime presence of symptoms. Zutshi, et al. [9] conducted a retrospective cohort study of patients who were diagnosed with tardive dyskinesia and discontinued their offending agent. Some of these patients received treatment for their TD, including switch to an atypical antipsychotic, beta blockers, benzodiazepines, or vesicular monoamine transporter 2 (VMAT2) inhibitors. Only $13.9 \%$ of these patients had resolution of $\mathrm{TD}$, and $2.8 \%$ experienced resolution of symptoms without any treatment. However, there was a general trend toward improvement, particularly in severe cases of TD, which showed a decrease in symptoms by a factor of 2.5 (AIMS score).

\section{Risk factors}

There are several demographic factors and co-morbid conditions which can lead to the development of TD. Advanced age is the most important factor, as shown in a study by Yassa, et al. [10]. This study showed that amongst geriatric patients who were initiated on antipsychotic treatment, $46 \%$ met criteria for TD after 5 years of treatment. Another significant risk factor the development of TD is female sex. It has been noted that the incidence of TD in elderly women being treated with antipsychotics is as high as 30\% [11]. History of alcohol abuse pre-disposes patients to development of TD [12]. Patients with mood disorders are also at risk to develop TD [13].

Pre-existing movement disorders may predispose patients to the development of tardive dyskinesia. Tenback, et al. [14] followed patients with and without extraparymidal symptoms (EPS) for 12 months. Patients with pre-existing EPS were more likely to develop TD (3\%) compared to patients without pre-existing EPS (1.6\%). It has also been shown that intention tremor is a risk factor for TD [12].

Polymorphisms in the genes which code for drug-metabolizing enzymes may also enhance a patient's risk for development of TD. Polymorphisms in the genes for the cytochrome oxidase pathway [15], catechol-O-methyl-transferase (COMT) and manganese superoxidedismutase (MsSOD) genes [16] have been noted to increase the risk for TD.

Phenylketonuria is another risk factor for the development of tardive dyskinesia [17]. The study showed evidence that men with tardive dyskinesia have a higher plasma concentration of phenylalanine after an oral phenylalanine challenge than controls. Glucose levels may also play a role in the development of symptoms. Animal studies have shown that dopamine receptor binding sensitivity is increased in diabetic rats [18] Given the proposed mechanism that tardive dyskinesia is caused by dopamine receptor super sensitivity, it is logical that impaired glucose tolerance would increase severity and incidence of TD symptoms. Schultz, et al. [19] found that, after controlling for age, elevated fasting glucose levels were correlated with higher AIMS scores in schizophrenic patients. However, there was no noted correlation between glucose tolerance after a $75 \mathrm{gm}$ glucose challenge and AIMS scores, suggesting that the baseline glucose levels, and not glucose tolerance, are implicated in the pathology of TD.

Low levels of brain-derived neurotrophic factor (BDNF) have been noted in patients with tardive dyskinesia, and BDNF serum concentrations have been found to be inversely related to AIMS scores in such patients [20]. This correlation is in line with other findings that BDNF is neuroprotective in the nigrostriatal pathway [21] and protective in neuronal damage mediated by glutamate [22]

Among schizophrenic patients receiving dopamine blocking agents, specific features of their presentation may predispose them to the development of TD. Severe negative symptoms have been shown to be a risk factor [23] and it also appears that the pattern of cognitive impairment in patients with TD and severe negative symptoms is similar to that of patients with basal ganglia pathology. Pantelis, et al. [24] assessed spatial working memory in patients with and without TD and found that those with TD performed worse on spatial working memory tasks, as well as delayed-matching-to-sample tasks compared to those without TD. Interestingly, this held true for patients with orofacial TD only, and patients with truncal TD did not perform worse compared to patients without TD.

The selection of antipsychotic agent, as well as type of administration appear to be of consequence. While second generation antipsychotics display a lower risk for tardive dyskinesia as a class, the comparative risk between medications in this group also varies. Correll, et al. [25] conducted a systematic review of 11 studies of patients treated with second generation antipsychotics [26-36]. In terms of risk by age, the annual incidence was found to be higher in geriatric patients $(5.3 \%)$ compared to the adult population (0.8\%). No tardive dyskinesia was noted in the study in children [31]. Adults being treated with haloperidol had an annual risk of 5.4\%. For second generation antipsychotics, the annual incidence of TD was as follows; olanzapine (0-0.5\%), risperidone $(0.6-0.7 \%$, including $0.7 \%$ in a study using long acting injectable risperdone), quetiapine (0.6-0.7\%), amisulpride (1.5\%), and ziprazidone (6.8\%). In the studies that had significant number of geriatric patients, the annual risk for TD were $2.7 \%$ for seroquel and between 2.6\%-13.4\% for risperidone. Tollefson, et al. [37] assessed patients receiving olanzapine and haloperidol for an average of 237 and 203 days, respectively. When the scores from the final two AIMS assessments for each group were analyzed, $1 \%$ of patients in the olanzapine group had met criteria for tardive dyskinesia, and $4.6 \%$ in the haloperidol group.

When tardive dyskinesia was first discovered, one common strategy to prevent the development of symptoms was to use antipsychotic medications intermittently. Such a regimen would result in less total antipsychotic exposure, which was thought to delay or prevent symptom development. However, such intermittent exposure may actually be counterproductive. Glenthoj [38] studied mice that were exposed to haloperidol for 6 months, either intermittently or continuously. It was noted that only the mice receiving intermittent doses, despite receiving a lower cumulative dose, developed TD symptoms following medication withdrawal. Harten, et al. [39] studied 
three factors in patients with and without tardive dyskinesia; number of interruptions in antipsychotic treatment, lifetime cumulative dose of antipsychotic medication (in chlorpromazine equivalents) and lifetime cumulative intake of anticholinergics). The study concluded that the most important factor among these was the total number of interruptions in treatment. The odds ratio was 3.29 for developing TD symptoms if the patient experienced more than 2 interruptions.

Risk for tardive dyskinesia in patients actively using antipsychotics appears to be dose dependent. Jeste, et al. [40] studied 330 geriatric patients (mean age 82.5) who were initiated on variable doses of risperidone for one year. Patients receiving less than $0.75 \mathrm{mg} /$ day did not meet the TD criteria, while $1.7 \%$ of those receiving $0.75 \mathrm{mg}-1.5 \mathrm{mg} /$ day and $5.5 \%$ of those receiving more than $1.5 \mathrm{mg} /$ day met the criteria.

\section{Management}

Several natural compounds have been assessed for possible treatment of tardive dyskinesia. Branch-chain amino acids (BCAAs) have been used in the management of tardive dyskinesia with some effect. BCAAs prevent aromatic amino acids from entering the central nervous system (CNS) via competition with aromatics in the bloodbrain barrier transport. In addition, ingestion of BCAAs decreases the plasma concentration of aromatic amino acids via increased protein synthesis $[41,42]$ Decreasing CNS concentrations of aromatic amino acids would be of benefit in tardive dyskinesia, as tyrosine, an aromatic amino acid, is the precursor of dopamine. Richardson, et al. [43] treated patients with BCAAs at a ratio of 3:3:4 (valine:isoleucine:leucine) or placebo for 3 weeks. Patients receiving BCAAs (dose of $222 \mathrm{mg} /$ day in divided doses) showed a $36.5 \%$ decrease in their score on the Simpson Abbreviated Dyskinesia Scale [44], compared to a 3.4\% increase in symptom severity in patients receiving placebo.

Given the possible role of oxidative stress in the pathophysiology of tardive dyskinesia, treatment with antioxidants have been attempted. Lerner, et al. [45] conducted a small crossover study of 15 patients in which patients alternated between receiving vitamin B 6 (titrated to $400 \mathrm{mg} /$ day) and placebo. Benefit was seen in both groups, showing an average improvement from baseline in the extrapyramidal symptom rating scale (ESRS) [46] of $68 \%$ and $38 \%$, respectively. Of note, there was no correlation between serum levels of vitamin B6 and symptom improvement, indicating that serum levels of pyridoxal-5'-phosphate are not directly related to tardive dyskinesia pathology.

As previously mentioned BDNF is neuroprotective and has been shown to be decreased in patients with tardive dyskinesia. Ginko biloba, a popular dietary supplement, has been shown to increase BDNF production in animal models, and thus may be beneficial to patients with tardive dyskinesia. Zhang, et al. [47] treated patients with a Ginko Biloba leaf extract EGb-761 at a dose of $80 \mathrm{mg}$ po three times per day in a 12-week study. Significant improvement in AIMS scores was seen in patients receiving EGb-761 at 6 and 12-week intervals compared to placebo. $30 \%$ or greater symptom improvement was seen in $52 \%$ of patients in the treatment group, compared to $5 \%$ of patients in the placebo group. Significant increase in serum BDNF levels were also seen in patients receiving the extract over those receiving placebo. Importantly, larger increases in serum BDNF levels correlated with larger improvements in AIMS scores in the treatment group.

Deep brain stimulation (DBS) may also be of benefit in patients with TD. Macerollo [48] showed that patients who were treated with DBS saw a mean improvement in AIMS scores of $62 \%$ after treatment.
Serotonin modulates dopamine release in the striatum, a region of the brain involved in movement, and could play a role in treatment of movement disorders. Clozapine, an antipsychotic with minimal risk of EPS and tardive dyskinesia, is noted to have a favorable 5-hydroxytryptamine-2(5HT2)/Dopamine 2(D2) binding ratio, as well has strong 5-hydroxytryptamine-3 (5-HT3) antagonism [49]. Ondansetron, a selective $5 \mathrm{HT} 3$ antagonist, was administered at $12 \mathrm{mg} /$ day to patients in an open label study by Sirota, et al. [50] AIMS mean severity score was noted to decrease by $78 \%$, in 13 out of 20 patients noting at least a $75 \%$ reduction in their AIMS scores. Also of note, this study showed a decrease in mean total positive and negative syndrome scale (PANSS) scores during treatment, from 95.2 at baseline to 69.6 after 12 weeks of treatment. This finding is in line with previous findings that ondansetron improves negative and cognitive symptoms in patients with schizophrenia [51]

Beta-blockers have been shown to decrease dopamine output from the striatum [52], and thus offer a novel pathway for the treatment of tardive dyskinesia. Hatcher-Martin, et al. [53] conducted a retrospective study of patients who had stopped neuroleptic treatment and had been initiated on propranolol (mean dose of $68.72 \mathrm{mg} /$ day). The study showed that $63.8 \%$ of patients improved with propranolol. Propranolol appears to be of added benefit in severe tardive dyskinesia. The odds of response to treatment were increased by 3.194 for every point increase in the severity of baseline tardive dyskinesia. The odds of response also increased by 1.09 for every month that the patient continued treatment, suggesting that symptom response to propranolol was gradual.

Clozapine, an atypical antipsychotic, not only has low rates of TD, but has been shown to benefit patients with TD who were previously on other antipsychotics. Spivak, et al. [54] studied patients who met criteria for TD and previously on another antipsychotic, switched to clozapine. At the conclusion of the 18-week trial, mean AIMS scores had improved by $74 \%$, with significant improvement in most patients by week 5 .

Benzodiazepines have been studied in the treatment of TD symptoms as well. Bhoopathi [55] reviewed 3 studies on the use of benzodiazepines in patients with TD, the conclusion of which showed little evidence of improvement in symptoms. However in our clinical experience, we have seen significant improvement in many cases.

A new drug valbenazine, a VMAT2 inhibitor, was the first drug to be approved for the treatment of tardive dyskinesia by the FDA in 2017. Hauser, et al. [56] studied patients receiving valbenazine at doses of $80 \mathrm{mg}$ daily, $40 \mathrm{mg}$ daily, and placebo for six weeks. At the study end, those who received $80 \mathrm{mg} /$ day had a mean decrease of 3.2 in their AIMS scores (effect size 0.90), while those receiving the lower $40 \mathrm{mg} /$ day or placebo saw a decrease of 1.9 and 0.1 , respectively. Differences from baseline AIMS scores were significant as early as week 2. Significant response, defined as an AIMS score improvement of at least 50\%, was seen in $40 \%$ of the group receiving $80 \mathrm{mg} /$ day, $23.8 \%$ of the $40 \mathrm{mg}$ /day group, and only $8.7 \%$ in the placebo group. In terms of safety, the most common side effects reported were somnolence (5.3\% in the treatment group vs 3.9 in the placebo group), akathisia (3.3\% vs $1.1 \%)$, and dry mouth $(3.3 \%$ vs $1.3 \%)$. There were no significant changes in physical examination, electrocardiogram, or routine laboratory tests. No difference in suicidal ideation was noted between groups. Following this study, an extension study of 42 weeks was conducted. In this extension study, patients received daily doses of either $40 \mathrm{mg}$ or $80 \mathrm{mg}$ of valbenazine. At the end of this study, both groups of patients saw improvement in AIMS scores, with a decrease in mean AIMS scores of 3 and 4.8, respectively. Following this treatment period, there was 
a four-week washout period. At the end of this washout period, both groups experienced a return to baseline in mean AIMS scores [57].

Deutetrabenazine, an isomer of tetrabenazine which is approved for treatment of Huntington's disease, was the second drug approved by the FDA for patients with tardive dyskinesia. While the exact mechanism of tetrabenazine is unknown, it is speculated to benefit patients with Huntington's disease and tardive dyskinesia by decreasing the amount of dopamine, as well as other monoamines, that are available for release in the presynaptic neuron. This is believed to be due to a decrease of dopamine which is packaged into the vesicles in the presynaptic neuron via blockade of vesicular transport. In a singleblind study, Ondo, et al. [58] studied patients with a mean dose of $57.9 \mathrm{mg} /$ day of tetrabenazine for 20 weeks, with a mean improvement of $54 \%$ (17.9 to 8.2) in their AIMS score. Deutetrabenazine was studied in a double-blind, placebo controlled trial in which patients received deutetrabenazine for 12 weeks. Patients receiving $24 \mathrm{mg}$ or $36 \mathrm{mg}$ daily saw a reduction in their mean AIMS scores of 3.2 and 3.3, respectively. While some akathisia was reported in the study, these side effects were generally mild, and drop-out rate for treatment groups were similar to placebo [59].

\section{Discussion}

Tardive dyskinesia presents a complex challenge to clinicians, and potentially life altering complications to patients. Assessing the incidence of TD is difficult, as many patients are not aware of their symptoms, and often a patient's symptoms may go unrecognized by their clinician. There is evidence that the syndrome is caused by upregulated postsynaptic doapamine receptors and oxidative neuronal damage, however a clear explanation of the pathology remains elusive. The risk of developing TD is greater in elderly patients, possibly due to increased sensitivity to dopamine blocking agents, or to naturally occurring chronic neuronal oxidative damage. Long term administration of antipsychotics is a key risk factor in the development of TD, however studies measuring lifetime antipsychotic use are dependent on accurate medication history for patients, which may be challenging. The expanded use of second generation antipsychotics has reduced the risk of tardive dyskinesia. A meta analysis of 11 studies showed the annual incidence of TD for patients being treated with haldol was $5.4 \%$ while the incidence was lower with atypical antipsychotics, including olanzapine (0-0.5\%), risperidone (0.6-0.7\%), quetiapine $(0.6-0.7 \%)$, amisulpride $(1.5 \%)$ [26-36]. It was noted that clozapine has a low risk for TD, however the incidence of other side effects such as agranulocytosis and the requirement for frequent laboratory monitoring limit clozapine use. While some natural products have been shown to offer some benefit, such as BCAA's and Vitamin B6, studies involving these agents are typically of small sample sizes, and resulted in only modest benefit. It was also noted that while patients taking vitamin B6 saw improvement in symptoms greater than those receiving placebo, there was no correlation between serum levels of vitamin B6 and symptom improvement. Two recently approved medications, valbenazine and deutetrabenazine, have been shown to improve symptoms in clinical trials. More long term studies are needed to evaluate the long term risks and benefits of these medications.

Our review is limited because it is an unstructured one. As more long term studies are conducted, a more cohesive assessment of these medications can be made.

\section{Conclusion}

Tardive dyskinesia is a very disabling and grotesque disorder, which did not have any FDA approved treatment until recently. The newly approved medications like valbenazine and tetrabenazine offer hope to these patients who otherwise would have to live with this condition causing potential social and clinical morbidity.

\section{Disclosures}

Both the authors do not have any conflicts of interest, financial or otherwise. Both authors have contributed significantly to the preparation of this manuscript.

The abstract of this manuscript has been accepted for a poster presentation at the 2019 May annual meeting of the American Psychiatric Association in San Francisco, California. ।

\section{References}

1. DeVaugh-Geiss J (1982) Tardive Dyskinesia and Related Involuntary Movement Disorders: The Long-term Effects of Antipsychotic Drugs. Boston: John Wright.

2. Burt DR, Creese I, Snyder SH (1977) Antischizophrenic drugs: Chronic treatment elevates dopamine receptor binding in the brain. Science 196: 326-328. [Crossref]

3. Creese I, Burt DR, Snyder SH (1997) Dopamine receptor binding enhancement accompanies lesion-induced behavioral supersensitivity. Science 197: 596-598. [Crossref]

4. Christensen E, Moller JE, Faurbye A (1970) Neuropathological investigation of 28 brains from patients with dyskinesia. Acta Psychiatr Scand 46: 14-23. [Crossref]

5. Bardgett ME, Wrona CT, Newcomer JW, Csernansky JG (1993) Subcortical excitatory amino acid levels after acute and subchronic administration of typical and atypical neuroleptics. Eur J Pharmacol 230: 245-250.

6. Olney JW (1990) Excitotoxic amino acids and neuropsychiatric disorders. Annu Rev Pharmacol Toxicol 30: 47-71.

7. Gouchuan T, Goff DC, Chang RW, Flood J, Baer L, et al. (1998) Markers of Glutamatergic Neurotransmission and Oxidative Stress Associated with Tardive Dyskinesia. Am J Psychiatry 155: 1207-1213.

8. Simpson GM, Angus JWS (1970) A rating scale for extrapyramidal side effects. Acta Psychiatr Scand Suppl 212: 11-19. [Crossref]

9. Zuutshi D, Cloud L, Factor SA (2014) Tardive Syndromes are Rarely Reversible after Discontinuing Dopamine Receptor Blocking Agents: Experience from a Universitybased Movement Disorder Clinic. Tremor and Other Hyperkinet Mov p. 4. [Crossref]

10. Yassa R, NAstase C, Dupont D, Thibeau M (1992) Tardive Dyskinesia in elderly psychiatric patients: a 5-year study. Am J Psychiatry 149: 1206-1211.

11. Jeste DV, Caligiuri MP, Paulsen JS, Heaton RK, Lacro JP, et al. (1995) Risk of tardive dyskinesia in older patients: a prospective longitudinal study of 266 outpatients. Arch Gen Psychiatry 52: 756-765.

12. Waln O, Jankovic J (2013) An update on tardive dyskinesia: from phenoenology to treatment. Tremor Other Hyperkinet Mov pp. 1-11. [Crossref]

13. Gardos G, Casey D (1984) Tardive Dyskinesia and Affective Disorders. American Psychiatric Press, Washington, DC.

14. Tenback DE, Van Harten PN, Slooff CJ, Van Os J (2006) Evidence That Early Extrapyramidal Symptoms Predict Later Tradive Dyskinesia: A Prospective Analysis of 10,000 Patients in the European Schizophrenia Outpatient Health Outcomes (SOHO) Study. Am J Psychiatry 163: 1438-1440.

15. Bertilsson L, Liisa Dahl M, Dalén P, Al-Shurbaji A (2002) "Molecular genetics of CYP2D6: clinical relevance with focus on psychotropic drugs." Br J Clin Pharmacol 53: 111-122. [Crossref]

16. Bakker PR, Van HArten PN, Van Os J (2008) "Antipsychotic-induced tardive dyskinesia and polymorphic variations in COMT, DRD2, CYP1A2 and MnSOD genes: a meta-analysis of pharmacogenetic interactions." Mol Psychiatr 13: 544.

17. Richardson MA, Haugland G, Pass R, Craig TJ (1986) The prevalence of tardive dyskinesia in a mentally retarded population. Psychopharmacol Bull 22: 243-249. [Crossref]

18. Lozovsky D, Saller C, Kopin I (1981) Dopamine receptor binding is increased in diabetic rats. Science 214: 1031-1032. [Crossref]

19. Schultz SK, Arndt SA, Ho B-C, Oliver SE, Andreasen NC (1999) Impaired Glucose Tolerance and Abnormal Movements in Patients with Schizophrenia. Am J Psychiatry 156: 640-642. 
20. Tan YL, Zhou DF, Zhang XY (2005) Decreased plasma brain-derived neurotrophic factor levels in schizophrenic patients with tardive dyskinesia: association with dyskinetic movements. Schizophr Res 74: 263-227.

21. Nishio T, Furukawa S, Akiguchi I, Sunohara N (1998) Medial nigral dopamine neurons have rich neurotrophin support in humans. Neuroreport 9: 2847-2851.

22. Cheng B, Mattson MP (1994) NT-3 and BDNF protect CNS neurons against metabolic/ excitotoxic insults. Brain Res 640: 56-67.

23. Liddle PF, Barnes TE, Speller J, Kibel D (1993) Negative symptoms as a risk factor for tardive dyskinesia in schizophrenia. Br J Psychiatry 163: 776-780.

24. Pantelis C, Stuart GW, Nelson HE, Robbins TW, Barnes TRE (2001) Spatial Working Memory Deficits in Schizophrenia: Relationship with Tardive Dyskinesia and Negative Symptoms. Am J Psychiatry 158: 1276-1285.

25. Correll C, Leucht S, Kane J (2004) Lower Risk of Tardive Dyskinesia Associated with Second-Generation Antipsychotics: A systemic Review of 1-Year Studies. Am J Psychiatry 161: 414-425. [Crossref]

26. Beasley CM, Dellva MA, Tamura RN, Morgenstern H, Glazer WM, et al. (1999) Randomised double-blind comparison of the incidence of tardive dyskinesia in patients with schizophrenia during long-term treatment with olanzapine or haloperidol. $\mathrm{Br} J$ Psychiatry 174: 23-30.

27. Arato M, O'Connor R, Meltzer HY (2002) A 1-year, double-blind, placebo-controlled trial of ziprasidone 40,80 and $160 \mathrm{mg}$ /day in chronic schizophrenia: the Ziprasidone Extended Use in Schizophrenia (ZEUS) Study. Int Clin Psychopharmacol 17: 207-215. [Crossref]

28. Csernansky JG, Mahmoud R, Brenner R (2002) A comparison of risperidone and haloperidol for the prevention of relapse in patients with schizophrenia. N Engl J Med 346: $16-22$.

29. Rein W, L'Héritier C (1999) Treatment-Emergent tardive dyskinesia in the long-term treatment of schizophrenia: a comparison of amisulpride and haloperidol (abstract). $J$ Eur Coll Neuropsychopharmacol 9: 282.

30. Chouinard G, Lasser R, Bossie C, Zhu Y, Gharabawi G (2002) Does a longacting atypical antipsychotic offer a low risk of tardive dyskinesia in patients with schizophrenia? in Proceedings of the 41st Annual Meeting of the American College of Neuropsychopharmacology. Tenn, ACNP, Nashville p. 356.

31. Turgay A, Binder C, Snyder R, Fisman S (2002) Long-term safety and efficacy of risperidone for the treatment of disruptive behavior disorders in children with subaverage IQs. Pediatrics 110: e34. [Crossref]

32. Glazer WM, Morgenstern H, Pultz JA, Yeung PP, Rak IW (1999) Incidence of persistent tardive dyskinesia may be lower with quetiapine treatment than previously reported with typical antipsychotics in patients with psychoses, in Proceedings of the 38th Annual Meeting of the American College of Neuropsychopharmacology. Tenn, ACNP, Nashville p. 271

33. Sanger TM, Grundy SL, Gibson PJ, Namjoshi MA, Greaney MG, et al. (2001) Long-term olanzapine therapy in the treatment of bipolar I disorder: an open-label continuation phase study. J Clin Psychiatry 62: 273-281.

34. Jeste DV, Okamoto A, Napolitano J, Kane JM, Martinez RA (2000) Low incidence of persistent tardive dyskinesia in elderly patients with dementia treated with risperidone. Am J Psychiatry 157: 1150-1155.

35. Davidson M, Harvey PD, Vervarcke J, Gagiano CA, De Hooge JD, et al. (2000) A longterm, multicenter, open-label study of risperidone in elderly patients with psychosis. Int $J$ Geriatr Psychiatry 15: 506-514.

36. Jeste DV, Glazer WM, Morgenstern H, Pultz JA, Yeung PP (1999) Rarity of persistent tardive dyskinesia with quetiapine: treatment of psychotic disorders in the elderly, in Proceedings of the 38th Annual Meeting of the American College of Neuropsychopharmacology. Tenn, ACNP, Nashville p. 142.

37. Tollefson GD, Beasley CM, Tamura RN, Tran PV, Potvin JH (1997) Blind, Controlled, Long Term Study of the Comparative Incidence of Treatment-Emergent Tardive Dyskinesia With Olanzapine or Haloperidol. Am J Psychiatry 154: 1248-1254.

38. Glenthoj B, Hemmingsen R (1989) Intermittent neuroleptic treatment induces long lasting abnormal mouthing in the rat. Eur J Pharmacol 164: 393-396.

39. Van Harten P, Hoek HW, Matroos GE, Koeter M, Khan RS (1998) Intermittent Neuroleptic Treatment and Risk of Tardive Dyskinesia: Curaco Extrapyramidal Syndromes Study III. Am J Psychiatry 155: 565-567.

40. Jeste DV, Okamoto A, Napolitano J, Kane JM, Martinez RA (2000) Low incidence of persistent Tardive Dyskinesia in Elderly Patients with Dementia Treated with Risperidone. Am J Psychiatry 157: 1150-1155.
41. Blomstrand E, Newsholme EA (1992) Effect of Branched-Chain amino acid supplementation on the exercise-induced change in aromatic amino acid concentration in human muscle. Acta Physiol Scand 146: 293-298.

42. Moldawer LL, Sakamoto A, Blackburn GL, Bistrain BR (1981) Alterations in protein kinetics produced by branched chain amino acid administration during infection and inflammation in Metabolism and Clinical Implications of Branched Chain Amino and Ketoacids. Edited by Walser M, Williamson JR. Elsevier-North Holland, New York pp. 533-539.

43. Richardson MA, Bevans ML, Read LL, Chao HM, Clelland JD, et al. (2003) Efficacy of the Branched-Chain Amino Acids in the Treatment of Tardive Dyskinesia in Men. Am J Psychiatry 160: 1117-1124.

44. Simpson GM, Lee JH, Zoubok B, Gardos G (1979) A rating scale for tardive dyskinesia. Psychopharmacology (Berl) 64: 171-179. [Crossref]

45. Lerner V, Miodownik C, Kaptsan A, Cohen H, Matar M, et al. (2001) Vitamin B6 in the Treatment of Tardive Dyskinesia: A Double-Blind, Placebo Controlled, Crossover Study. Am J Psychiatry 158: 1511-1514.

46. Chouinard G, Ross-Chouinard A, Annable L, Jones B (1980) Extrapyramidal Symptom Rating Scale (abstract). Can J Neurol Sci 7: 233.

47. Zhang XY, Zhang WF, Zhou DF, Chen DC, Xiu MH, et al. (2012) Brain-Derived Neurotrophic Factor Levels and Its Val66Met Polymorphism Predict Tardive Dyskinesia Treatment Response to Ginko Biloba. Biol Psychiatry 72: 700-706. [Crossref]

48. Macerollo A, Deuschl G (2018) Deep brain stimulation for tardive syndromes: Systematic review and meta-analysis. J Neurol Sci 389: 55-60.

49. Hoyer D, Clarke DE, Fozard JR, Hartig PR, Martin GR, et al. (1994) Internationa Union of Pharmacology classification of receptors for 5-hydroxy-tryptamine (serotonin). Pharmacol Rev 46: 157-203.

50. Sirota P, Mosheva T, Shabtay H, Giladi N, Korczyn AD (2000) Use of Selective Serotonin 3 Receptor Antagonist Ondansetron in the Treatment of Neuroleptic-Induced Tardive Dyskinesia. Am J Psychiatry 157: 287-289.

51. Zhang ZJ, Khang WH, Li Q, Wang XY, Yao SM, et al. (2006) Beneficial effects of Ondansetron as an adjunct to haloperidol for chronic, treatment-resistant schizophrenia: a double-blind, randomized, placebo-controlled study. Schizophr Res 88: 102-110.

52. Bhide N, Lindenbach D, Barnum CJ, George JA, Surrena MA, et al. (2015) Effects of the beta-adrenergic receptor antagonist Propranolol on dyskinesia and L-DOPAinduced striatal DA efflus in the hemi-parkinsonian rat. $J$ Neurochem 134: 222-232. [Crossref]

53. Hatcher-Martin JM, Armstrong KA, Scorr LM, Factor SA (2016) Propranolol therapy for Tardive Dyskinesia: A retrospective examination. Parkinsonism Relat Disord $\mathrm{p}$. 32. [Crossref]

54. Christensen E, Moller JE, Faurbye A (1970) Neuropathological investigation of 28 brains from patients with dyskinesia. Acta Psychiatr Scand 46: 14-23. [Crossref]

55. Bardgett ME, Wrona CT, Newcomer JW, Csernansky JG (1993) Subcortical excitatory amino acid levels after acute and subchronic administration of typical and atypical neuroleptics. Eur J Pharmacol 230: 245-250.

56. Hauser RA, Factor SA, Marder SR, Knesevich MA, Ramirez PM, et al. (2017) Kinect 3: A phase 3 Randomized, Double-Blind, Placebo-Controlled Trial of Valbenazine for Tardive Dyskinesia. Am J Psychiatry 174: 467-484. [Crossref]

57. Factor SA, Remington G, Comella CL, Correll C, Burke J, et al. (2017) The effects of valbenazine in participants with tardive dyskinesia: results of the 1-year KINECT 3 extension study. J Clin Psychiatry 78: 1344-1350.

58. Ondo WG, Hanna PA, Jankovic J (1999) Tetrabenazine Treatment for Tardive Dyskinesia: Assessment by Randomized Videotape Protocol. Am J Psychiatry 156: 1279-1281. [Crossref]

59. Anderson KE, Stamler D, Davis MD, Factor SA, Hauser RA, et al. (2017) Deutetrabenazine for treatment of involuntary movements in patients with tardive dyskinesia (AIM-TD): a double-blind, randomised, placebo-controlled, phase 3 trial. Lancet Psychiatry 4: 595-604. [Crossref]

Copyright: (C2019 Madhusoodanan S. This is an open-access article distributed under the terms of the Creative Commons Attribution License, which permits unrestricted use, distribution, and reproduction in any medium, provided the original author and source are credited. 